\title{
A Hierarchical Upscaling Method for Predicting Strength of Materials under Thermal, Radiation and Mechanical loading - Irradiation Strengthening Mechanisms in Stainless Steels
}

\section{Fuel Cycle Research \& Development}




\section{DISCLAIMER}

This information was prepared as an account of work sponsored by an agency of the U.S. Government. Neither the U.S. Government nor any agency thereof, nor any of their employees, makes any warranty, expressed or implied, or assumes any legal liability or responsibility for the accuracy, completeness, or usefulness, of any information, apparatus, product, or process disclosed, or represents that its use would not infringe privately owned rights. References herein to any specific commercial product, process, or service by trade name, trade mark, manufacturer, or otherwise, does not necessarily constitute or imply its endorsement, recommendation, or favoring by the U.S. Government or any agency thereof. The views and opinions of authors expressed herein do not necessarily state or reflect those of the U.S. Government or any agency thereof. 


\section{Reviewed by:}

Acting Director, Fuel Cycle Research and

Development

Robert Price

Date

\section{Concurred by:}

Director, Advanced Fuel Cycle Initiative

Technical Integration Office

\section{Approved by:}

Deputy Assistant Secretary, Fuel Cycle

Management

(AFCI Program Manager)

Paul Lisowski

Date 


\section{SUMMARY}

Stainless steels based on iron-chromium-nickel (Fe-Cr-Ni) alloys are the most popular structural materials used in reactors. High energy particle irradiation in these types of polycrystalline structural materials usually produces irradiation hardening and embrittlement. The development of predictive capability for the influence of irradiation on mechanical behavior is important in materials design for next-generation reactors. Irradiation hardening is related to structural information crossing different length scales, such as composition, dislocation, and crystal orientation distribution. To predict effective hardening, the influence factors along different length scales should be considered. A multiscale approach was implemented in this work to predict irradiation hardening of Fe based structural materials.

Three length scales are involved in this hierarchical upscaling method: nanometer, micrometer, and millimeter. In the microscale, molecular dynamics (MD) was used to predict the edge dislocation mobility in body centered cubic (bcc) $\mathrm{Fe}$ and its $\mathrm{Ni}$ and $\mathrm{Cr}$ alloys. On the mesoscale, dislocation dynamics (DD) models were used to predict the critical resolved shear stress (CRSS) from the evolution of local dislocation and defects. In the macroscale, a viscoplastic self-consistent (VPSC) model was applied to predict the irradiation hardening in samples with changes in texture.

The effects of defect density and texture were investigated. Simulated evolution of yield strength with irradiation agrees well with the experimental data on irradiation strengthening of stainless steel 304L, 316L, and T91. The hierarchical upscaling method developed in this project can provide a guidance tool to evaluate performance of structural materials for next-generation nuclear reactors. Combined with other tools developed in the Nuclear Energy Advanced Modeling and Simulation (NEAMS) program, the models developed will have more impact in improving the reliability of current reactors and affordability of new reactors. 


\section{CONTENTS}

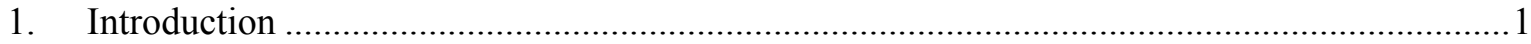

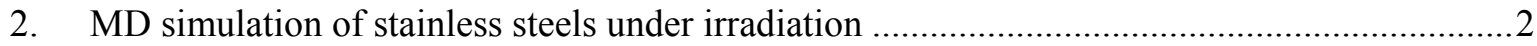

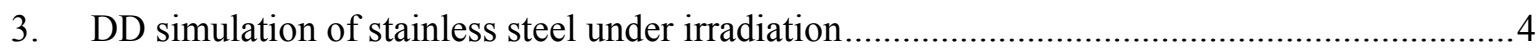

4. Irradiation strengthening simulation using viscoplastic self-consistent model ........................5

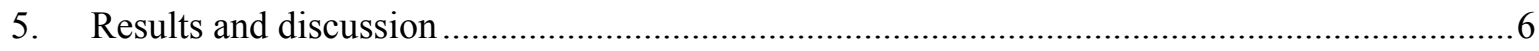

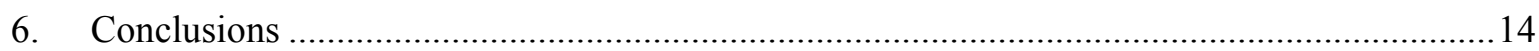

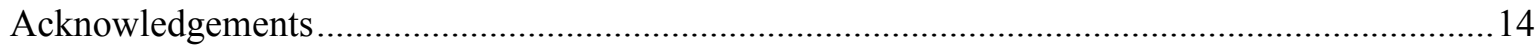

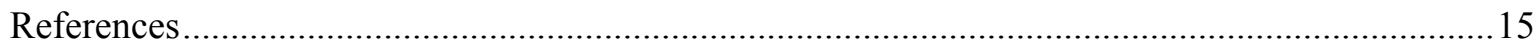




\section{LIST OF FIGURES}

Figure 1. The configuration used in mobility simulation using MD.

Figure 2. Edge dislocation mobility of four Fe-Cr-Ni alloys and one control Fe material at

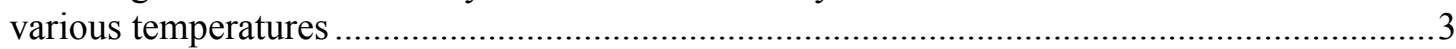

Figure 3. CRSS of Fe-Ni alloys as a function of Ni concentration..............................................

Figure 4.a) A snapshot of dislocations in the simulation cell during deformation (colors correspond dislocation type; dots are FS loops). b) An enlarged section showing dislocations pinned by the defects.

Figure 5. Predicted stress-strain curves from dislocation dynamics for various initial defect density

Figure 6. Resolved shear stress under different defect density predicted by dislocation dynamics models. Black line is fit to exponential function from the predicted results.

Figure 7. (100), (110), and (111) pole figures of irradiated alpha-iron with defect density of $10^{23}$ $\mathrm{m}^{-3}$ : (a) random sample, (b) uniaxial extended to a strain of $50 \%$, and (c) uniaxial compressed to a strain of $50 \%$.

Figure 8. Predicted stress strain curve of irradiated alpha-iron with defect density of $10^{23} \mathrm{~m}^{-3}$ under uniaxial tension and compression.

Figure 9. Predicted yield strength as a function of defect density in alpha-iron with different microstructure.

Figure 10. Variation of the yield stress with irradiation dose for stainless steel 304L (Fig.10a), 316L (Fig. 10b) and modified T91 (Fig. 10c). Experimental data (open square symbols) are compared with simulation results of filled symbols. 
Hierarchical Upscaling Method for Irradiation Strengthening Mechanisms in Stainless Steels

\section{LIST OF TABLES}

Table 1. Mobility of an edge dislocation inside $\mathrm{Fe}-\mathrm{Ni}-\mathrm{Cr}$ alloys at various temperatures..................2

Table 2: Materials parameters used in multiscale modeling the irradiation strengthening...............14 


\section{ACRONYMS}

$\begin{array}{ll}\text { bcc } & \text { body-centered cubic } \\ \text { Cr } & \text { chromium } \\ \text { CRSS } & \text { critical resolved shear stress } \\ \text { DD } & \text { dislocation dynamics } \\ \text { DOE } & \text { U.S. Department of Energy } \\ \text { fcc } & \text { face-centered cubic } \\ \text { Fe } & \text { iron } \\ \text { FS } & \text { Frank sessile } \\ \text { MD } & \text { molecular dynamics } \\ \text { Ni } & \text { nickel } \\ \text { PNNL } & \text { Pacific Northwest National Laboratory } \\ \text { R\&D } & \text { research and development } \\ \text { VPSC } & \text { viscoplastic self-consistent }\end{array}$




\section{FUEL CYCLE R\&D PROGRAM Multiscale Models for Irradiation Strengthening Mechanisms in Stainless Steel}

\section{INTRODUCTION}

Rapidly increasing demand of energy and an unstable supply of fossil fuels are reviving nuclear power [1-2]. Development of solar and wind energy is promising, but they fall short of substituting fossil fuels in the near term and on a large scale. The only mature energy technology to replace fossil fuels is nuclear energy, which now supplies $78 \%$ of the electricity in France. The current challenges in nuclear energy are safety and waste storage. One of the solutions is to develop new high-performance structural materials that will retain excellent thermal mechanical properties under long-term irradiation and other hostile environments. This vision is reflected in the U.S. Department of Energy's (DOE) nuclear energy research and development $(\mathrm{R} \& \mathrm{D})$ programs [1-2].

Under irradiation, atoms in structural materials will be displaced from their lattice positions. The irradiation damage creates different microstructure changes, such as void growth, dislocation structure evolution, defect clusters, helium bubbles, etc. The new microstructures exhibit degraded mechanical properties, such as irradiation hardening and embrittlement [3-4]. Defect density and cluster size evolves during irradiation. The challenge to simulate mechanical behavior with defect density and cluster size attracts scientists from varied communities, and different approaches have been considered. At the macroscopic scale, defects generally are treated as barriers to the motion of dislocations. According to the assumption of the treatment of defects, they are divided into two classes: 1) strong obstacles model and 2) weak obstacles model. In the first theory, the defects directly intersect the dislocation glide plane and increase the planar geometric spacing. In the second theory, the defects induce the increase of effective interparticle spacing due to less extensive dislocation bowing prior to obstacle breakaway. These models proposed polynomial or power laws to describe the relationship between the yield strength and defect cluster properties, which usually are represented by size and density. Parameters of these models are obtained by fitting in the experimental data.

Deo et al. (2008)[1] modeled the yield stress dependence in ferritic steels on the irradiation dose by passing the critical resolved shear stress (CRSS) simulated from these barrier hardening models directly to a higher-scale model, the viscoplastic self-consistent (VPSC) model. Defect cluster size and density were assumed proportional to the square root of the irradiation dose.

Macroscopic modeling is useful in describing the irradiation effect on selected materials under specific environments. These phenomenal models are not enough to guide materials performance evaluation under irradiation, which includes newly developed materials under different chemical and irradiation environments. Understanding the irradiation effect from the basic science point of view is necessary to predict the effects of irradiation on microstructure and properties, an inherently multiscale phenomenon. Most current simulation efforts are focused on the microscopic scale to understand the processes of atomic dynamics, molecular dynamics (MD), and dislocation dynamics (DD) models that have been developed to predict the nucleation, development, and movement of dislocation in irradiated alpha-iron [5-7]. Mechanisms of dislocation development in different chemical environments have been investigated. Defect cluster formation and growth have been studied. Further, the interaction between the defect clusters and dislocation has been studied. Some multiscale approaches have been undertaken to 
bridge the DD and continuum crystal plasticity to predict the mechanical behavior in irradiated structural materials [8-11]. The scale-dependent effects of grain size on dislocation movement and yield stress have been predicted and agree with the experimental observation. Usually, the microstructures are assumed homogenous, and the components, in this case, crystals, are considered isotropic. Herein, we take another multiscale approach to perform a parametric study on effects of texture by bridging the MD, DD, and viscoplastic homogenization models.

\section{MD SIMULATION OF STAINLESS STEELS UNDER IRRADIATION}

Edge dislocation mobility in body-centered cubic (bcc) iron (Fe) and its nickel (Ni) and chromium (Cr) alloys was simulated using MD. The configuration of atomic spatial model is illustrated in Figure 1. The edge dislocation is shown in the middle of the simulation box, and the arrows depict the loading direction.

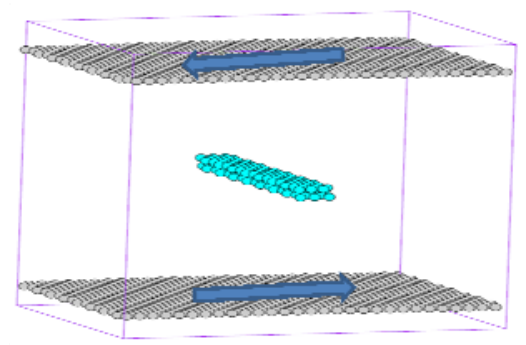

Figure 1. The configuration used in mobility simulation using MD

Simulated dislocation mobility under different working temperature and chemical compositions of $\mathrm{Ni}$ and $\mathrm{Cr}$ is listed in Table 1.

Table 1. Mobility of an edge dislocation inside $\mathrm{Fe}-\mathrm{Ni}$-Cr alloys at various temperatures.

\begin{tabular}{|c|c|c|c|c|c|c|c|}
\hline $\begin{array}{c}\text { Temp } \\
\text { Struct. }\end{array}$ & $10 \mathrm{~K}$ & $50 \mathrm{~K}$ & $100 \mathrm{~K}$ & $150 \mathrm{~K}$ & $200 \mathrm{~K}$ & $250 \mathrm{~K}$ & $300 \mathrm{~K}$ \\
\hline$\alpha-\mathrm{Fe}$ & $3.55 \mathrm{E}+03$ & $2.93 \mathrm{E}+03$ & $2.61 \mathrm{E}+03$ & $2.50 \mathrm{E}+03$ & $2.43 \mathrm{E}+03$ & $2.38 \mathrm{E}+03$ & $2.38 \mathrm{E}+03$ \\
\hline $\mathrm{Fe}-5 \% \mathrm{Ni}$ & $4.31 \mathrm{E}+03$ & $4.08 \mathrm{E}+03$ & $4.02 \mathrm{E}+03$ & $3.68 \mathrm{E}+03$ & $3.73 \mathrm{E}+03$ & $3.79 \mathrm{E}+03$ & $2.79 \mathrm{E}+03$ \\
\hline $\mathrm{Fe}-10 \% \mathrm{Ni}$ & $5.05 \mathrm{E}+03$ & $4.37 \mathrm{E}+03$ & $4.13 \mathrm{E}+03$ & $4.07 \mathrm{E}+03$ & $4.07 \mathrm{E}+03$ & $4.31 \mathrm{E}+03$ & $3.94 \mathrm{E}+03$ \\
\hline $\mathrm{Fe}-15 \% \mathrm{Ni}$ & $5.43 \mathrm{E}+03$ & $4.41 \mathrm{E}+03$ & $4.39 \mathrm{E}+03$ & $4.85 \mathrm{E}+03$ & $5.21 \mathrm{E}+03$ & $5.56 \mathrm{E}+03$ & $5.00 \mathrm{E}+03$ \\
\hline $\mathrm{Fe}-20 \% \mathrm{Ni}$ & $5.99 \mathrm{E}+03$ & $4.52 \mathrm{E}+03$ & $4.65 \mathrm{E}+03$ & $5.03 \mathrm{E}+03$ & $5.29 \mathrm{E}+03$ & $5.29 \mathrm{E}+03$ & $5.18 \mathrm{E}+03$ \\
\hline $\mathrm{Fe}-5 \% \mathrm{Cr}$ & $3.48 \mathrm{E}+03$ & $2.82 \mathrm{E}+03$ & $2.67 \mathrm{E}+03$ & $2.55 \mathrm{E}+03$ & $2.56 \mathrm{E}+03$ & $2.60 \mathrm{E}+03$ & $2.71 \mathrm{E}+03$ \\
\hline $\mathrm{Fe}-10 \% \mathrm{Cr}$ & $3.57 \mathrm{E}+03$ & $3.08 \mathrm{E}+03$ & $2.95 \mathrm{E}+03$ & $3.07 \mathrm{E}+03$ & $3.12 \mathrm{E}+03$ & $3.18 \mathrm{E}+03$ & $3.15 \mathrm{E}+03$ \\
\hline $\mathrm{Fe}-15 \% \mathrm{Cr}$ & $3.68 \mathrm{E}+03$ & $3.09 \mathrm{E}+03$ & $2.92 \mathrm{E}+03$ & $2.72 \mathrm{E}+03$ & $2.84 \mathrm{E}+03$ & $2.78 \mathrm{E}+03$ & $3.13 \mathrm{E}+03$ \\
\hline $\mathrm{Fe}-20 \% \mathrm{Cr}$ & $2.98 \mathrm{E}+03$ & $2.73 \mathrm{E}+03$ & $2.59 \mathrm{E}+03$ & $2.69 \mathrm{E}+03$ & $2.82 \mathrm{E}+03$ & $2.83 \mathrm{E}+03$ & $3.09 \mathrm{E}+03$ \\
\hline
\end{tabular}


For a clearer demonstration, the evolution of mobility along with temperature of four Fe-Cr-Ni alloys and one control $\alpha-\mathrm{Fe}$ is illustrated in Figure 2. The temperature range investigated was from $10 \mathrm{~K}$ to $300 \mathrm{~K}$. The results reveal that the dislocation mobility decreases as the temperature increases. The decrease is not linear and generally saturates at a temperature of about $200 \mathrm{~K}$. After passing the saturation temperature, the mobility remains relatively constant with temperature. The effect of concentration of Ni and $\mathrm{Cr}$ was also investigated. The addition of Ni results in an increase in the dislocation mobility, while the addition of $\mathrm{Cr}$ does not appear to affect the mobility considerably. This may be attributed to the type of the solute (fcc Ni vs. bcc $\mathrm{Cr}$ ) since both the atomic masses of $\mathrm{Ni}$ and $\mathrm{Cr}$ are comparable to that of Fe.

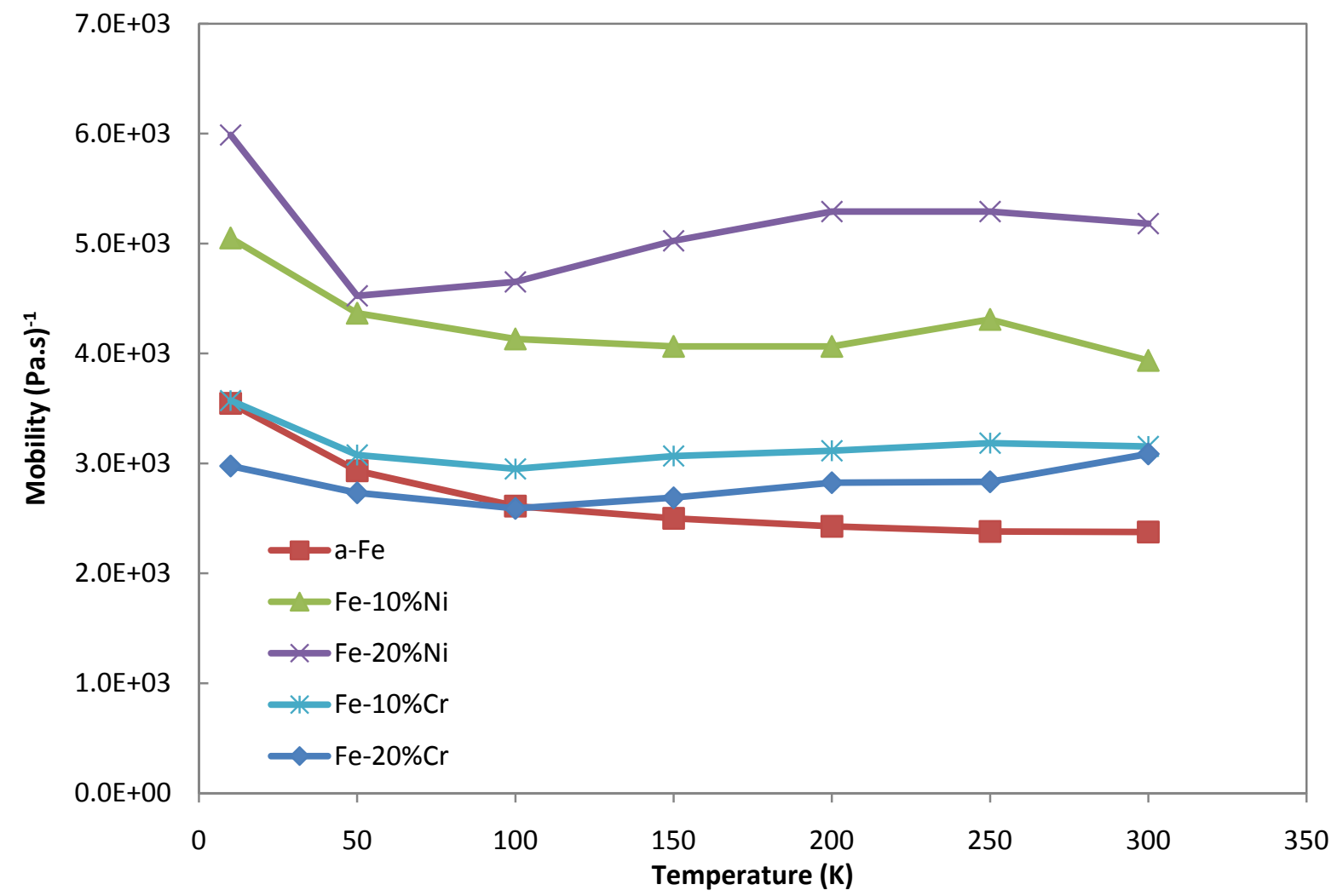

Figure 2. Edge dislocation mobility of four $\mathrm{Fe}-\mathrm{Cr}-\mathrm{Ni}$ alloys and one control $\mathrm{Fe}$ material at various temperatures

The effect of the Ni concentration on the CRSS of an edge dislocation to pass through a void was also investigated. The results reveal that the addition of Ni increases the critical stress, an effect that is more pronounced as the Ni concentration reaches $20 \%$. The first results for a void of $2 \mathrm{~nm}$ in diameter (shown in Figure 3) reveal a logarithmic dependence of the stress from the Ni concentration. However, more simulations are necessary to clarify this trend, as well as the effect of the void size and void-void distance.

To simulate evolution of dislocation during mechanical deformation, the simulation results obtained from MD are fed into the DD software package. The mechanical deformation response of single crystal will be investigated in the next section. 


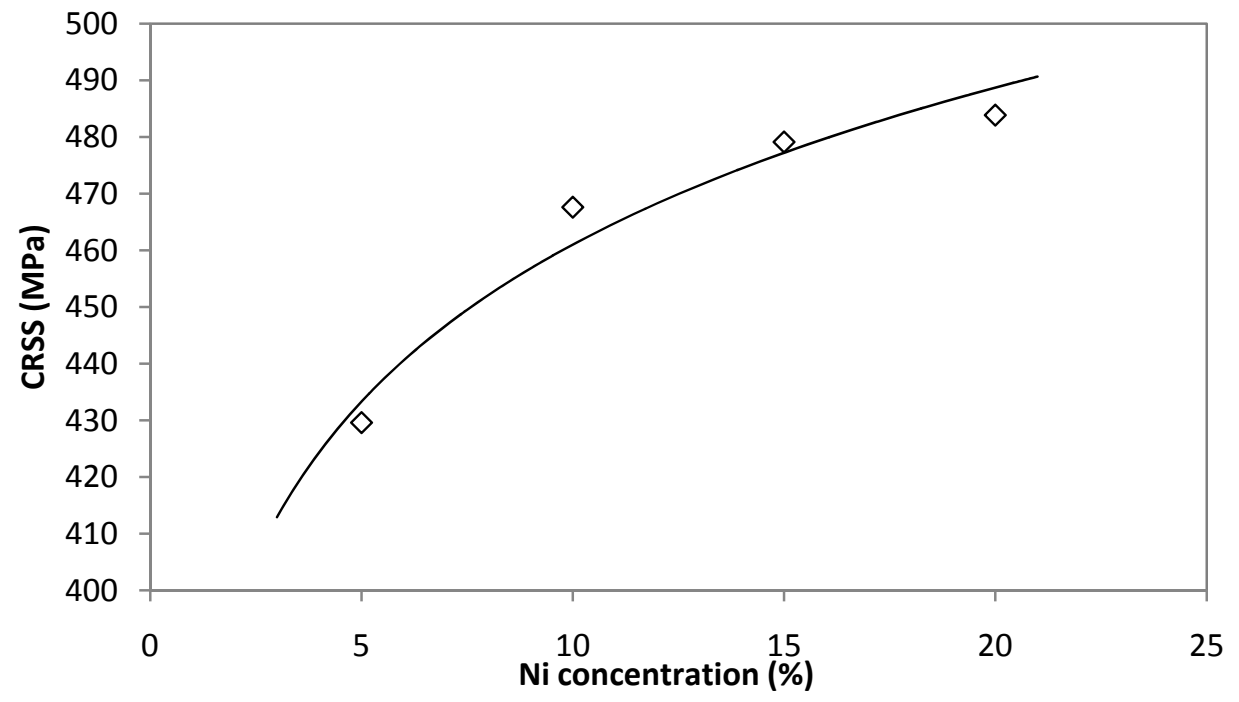

Figure 3. CRSS of Fe-Ni alloys as a function of Ni concentration

\section{DD SIMULATION OF STAINLESS STEEL UNDER IRRADIATION}

The DD method is a tool to explicitly model dislocation motion and dislocation-defect interactions based on the fundamentals of dislocation theory. It is used, among other things, to predict the stressstrain curve and the evolution of dislocation structure and their dependence on variables such as temperature, strain rate, defects, particles, etc. A complete description of the DD method and its extension to dislocations in heterogeneous media can be found in the article by Zbib and de la Rubia (2002) [2]. According to the theory, the motion of a dislocation segment is governed by a Newtoniantype equation of motion with a driving force that consists of a number of components: Peierls lattice friction forces, dislocation-dislocation interaction forces through their stress fields, forces due to externally applied forces, osmotic climb forces, and images forces due to free surfaces and/or from difference in elastic moduli for dislocations in heterogeneous media and near interfaces. In the case of irradiation damage, dislocations also interact with defect clusters whose density in bcc metals can be on the order of $10^{24} \mathrm{~m}^{-3}$ for a variety of neutron-irradiated metals near room temperature. In bcc metals, defects are Frank Sessile (FS) dislocation loops of the type $\{100\}$, Burgers vector of the type $<100>$, and the radius varies between $1 \mathrm{~nm}$ to $3 \mathrm{~nm}$.

In DD, the elastic interaction and short-range interaction between dislocations and FS loops is computed explicitly based on the work of Khraishi et al. (2002) [3], which was originally developed for fcc systems. Each defect possesses its own stress field, which contributes to the Peach-Koehler force, impeding the motion of the dislocation and contributing to irradiation hardening. 
In the present work, we investigate the effect of irradiation hardening in bcc. The goal is to predict the stress-strain curve and the CRSS as a function of defect density, which then is used in the crystal plasticity model.

The DD simulation box is a single crystal $2 \times 2 \times 6 \mu \mathrm{m}^{3}$ cube that contains an initial density of FrankRead sources distributed randomly on the primary $\{110\}$ slip planes. In the simulations, periodic boundary conditions were imposed. The cell is loaded uniaxially in tension with a constant strain rate of $100 / \mathrm{s}$. The effect of irradiation is accounted for by mapping into the DD box a spatial distribution of FS loops with density ranging from $10^{20} \mathrm{~m}^{-3}$ to $10^{24} \mathrm{~m}^{-3}$. The loops are $1 \mathrm{~nm}$ to $3 \mathrm{~nm}$ in radius, and the radius is randomly generated to fall within the specified interval. The model also generates Frank-Read sources, represented as finite dislocation segments pinned at ends, lying on $\{110\}$ glide planes, and Burgers vectors of the type $<111>$.

In the DD simulations, the required material parameters are: the shear modulus, $\mathrm{G}$; the Poisson $v$; the dislocation mobility, $M$; the Burgers vector magnitude, $b$. For alpha-iron at $\mathrm{T}=300 \mathrm{~K}$, we use $\mathrm{G}=82.2$ $\mathrm{GPa}, \mathrm{M}=103 / \mathrm{Pa} \cdot \mathrm{s}$ for edge mixed dislocation, $\mathrm{M}=0.1 / \mathrm{Pa} \cdot \mathrm{s}$ for screw dislocation [4], $b=0.2482 \mathrm{~nm}$, and $v=0.289$.

\section{IRRADIATION STRENGTHENING SIMULATION USING VISCOPLASTIC SELF-CONSISTENT MODEL}

After the DD model predicts CRSS, it is fed into the upper scale polycrystalline model to predict the effective irradiation hardening and deformation behavior. At this scale, a VPSC model, developed by Tome and Lebensohn [5-6], was applied to predict the evolution of hardening and texture evolution associated with plastic forming. Along with the macroscopic stress-strain response, this model also accounts for hardening, reorientation, and shape change of individual grains.

In this model, plastic deformation is attributed to slip systems characterized by a slip plane normal vector, $\boldsymbol{n}$, and a Burgers vector, $\boldsymbol{b}$, or twinning systems characterized by a twinning plane normal vector, $\boldsymbol{n}$, and twin shear direction, $\boldsymbol{b}$.

The CRSS $\tau^{\alpha}$ is the projection of deviatoric Cauchy stress, $S$, on the system $\alpha$, using the Schmid tensor, $\mathrm{P}^{\alpha}$ :

$$
\tau^{\alpha}=P^{\alpha} \cdot S=\frac{1}{2}\left(n_{i} b_{j}+n_{j} b_{i}\right) \cdot S \quad .
$$

The corresponding plastic strain rate for the system $\alpha, \mathrm{D}^{\alpha}$ also is the projection of shear rate $\dot{\gamma}^{\alpha}$, using $\mathrm{P} \alpha$ :

$$
\mathrm{D}^{\alpha}=\mathrm{P}^{\alpha} \cdot \dot{\gamma}^{\alpha}=\frac{1}{2}\left(\mathrm{n}_{\mathrm{i}} \mathrm{b}_{\mathrm{j}}+\mathrm{n}_{\mathrm{j}} \mathrm{b}_{\mathrm{i}}\right) \cdot \dot{\gamma}^{\alpha} .
$$

A classical viscoplastic power law is used to describe the relationship between $\dot{\gamma}^{\alpha}$ and the corresponding resolved shear stress $\tau \alpha$ CRSS:

$$
\dot{\gamma}^{\alpha}=\dot{\gamma}^{0}\left(\frac{\tau^{\alpha}}{\mathrm{g}^{\alpha}}\right)^{\mathrm{n}}
$$

where $\dot{\gamma}^{0}$ is a reference shear rate, which often is assumed to be the same for all systems.; $\mathrm{n}$ is the inverse of strain rate sensitivity coefficient, and $\mathrm{g}^{\alpha}$ is the CRSS of system $\alpha$ evolving with strain hardening summed over $\beta$ systems, according to Kalidindi [7]: 


$$
\dot{g}^{\alpha}=\sum_{\beta} H^{\alpha \beta}\left|\dot{\gamma}^{\beta}\right|=\sum_{\beta}\left(q^{\alpha \beta} h_{0}\left[1-\frac{g^{\alpha}}{g_{0}}\right]^{\mathrm{a}}\right)\left|\dot{\gamma}^{\beta}\right| .
$$

Here, $\mathrm{H}^{\alpha \beta}$, the strain-hardening matrix on the system $\alpha$, is due to a shearing on another system $\beta$, which is a function of latent hardening matrix $\mathrm{q}^{\alpha \beta}$, the initial hardening slope $\mathrm{h}_{0}$, the stress level for the hardening saturation $\mathrm{g}_{0}$, and rate-hardening power parameter $\mathrm{a}$.

The strain rate for each single crystal, D, is a sum carried over all of the systems:

$$
\mathrm{D}=\dot{\gamma}^{0} \sum_{\alpha}\left(\frac{\tau^{\alpha}}{\mathrm{g}^{\alpha}}\right)^{\mathrm{n}}=\dot{\gamma}^{0} \sum_{\alpha}\left(\frac{1}{\mathrm{~g}^{\alpha}}\left(\frac{\tau^{\alpha}}{\mathrm{g}^{\alpha}}\right)^{\mathrm{n}-1}\right) \mathrm{P}^{\alpha} \cdot \mathrm{S}=\mathrm{M}^{\mathrm{sec}} \mathrm{S} .
$$

Rewritten in a pseudo-linear format, the slope, $\mathrm{M}^{\mathrm{sec}}$, is the secant viscoplastic compliance modulus of the grain, which still is a function of the deviatoric Cauchy stress, $\mathrm{S}$.

At the polycrystalline level, the whole microstructure is treated as ellipsoidal viscoplastic single crystal inclusions embedded in a homogeneous equivalent medium, which represents the effective environment. The macroscopic behavior can be described by a similar constitutive law:

$$
\overline{\mathrm{D}}=\overline{\mathrm{M}} \overline{\mathrm{S}}
$$

in which $\overline{\mathrm{D}}, \overline{\mathrm{M}}$, and $\overline{\mathrm{S}}$ represent macroscopic strain rate, macroscopic compliance, and macroscopic deviatoric Cauchy stress, respectively. The same compliance also applies on the homogenous equivalent medium.

An interaction equation was introduced to solve the inhomogeneity, equilibrium equation for inhomogeneous ellipsoidal single crystals embedded in a homogeneous medium:

$$
(\mathrm{D}-\overline{\mathrm{D}})=\widetilde{\mathrm{M}}(\sigma-\bar{\sigma}) \text {, }
$$

where $\widetilde{\mathrm{M}}$ is the interaction matrix defined as:

$$
\widetilde{\mathrm{M}}=\mathrm{n}^{\text {eff }}(\mathrm{I}-\mathrm{E})^{-1} \mathrm{E} \overline{\mathrm{M}} \text {. }
$$

Here, $\mathrm{E}$ is the Eshelby tensor, a homogenous function of grain shape. The parameter $\mathrm{n}^{\text {eff }}$ tunes the strength of the coupling between the stress deviations and the associated strain rate deviations. It depends on the used constitutive assumption. $I$ is the fourth order identity tensor. This interaction law provides a solution for the deviation of the strain rate in the grain with respect to the macroscopic average.

\section{RESULTS AND DISCUSSION}

A series of DD simulations were performed. Within this section, we provide results relevant to the polycrystalline plasticity model previously discussed. Figure 4 shows the dislocation structure after a certain amount of strain in the presence of FS loops. 


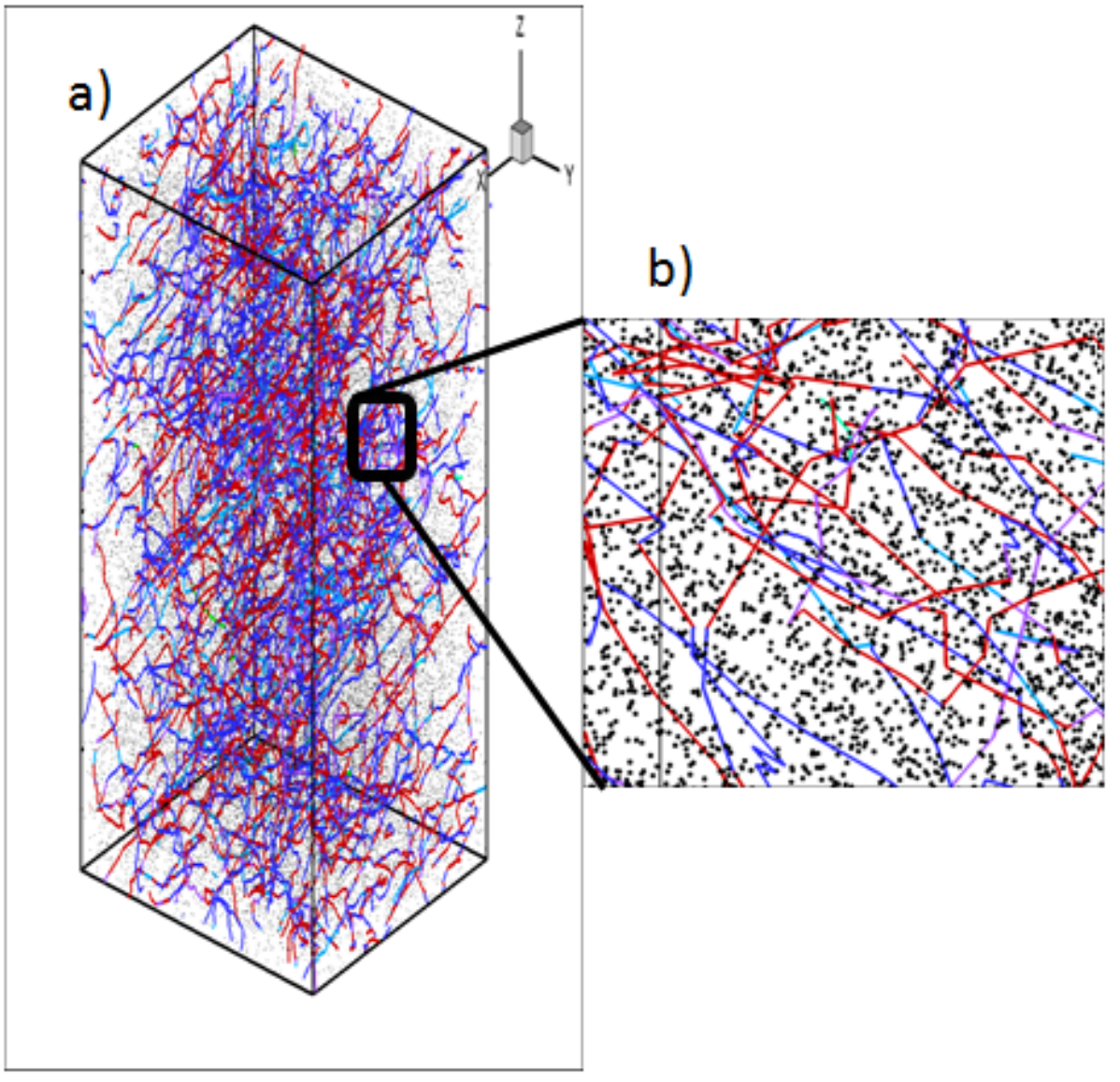

Figure 4.a) A snapshot of dislocations in the simulation cell during deformation (colors correspond dislocation type; dots are FS loops). b) An enlarged section showing dislocations pinned by the defects.

The predicted stress strain curves are shown in Figure 5. The figure demonstrates strong dependence of deformation behavior on the initial defect density. Initially, the stress increases linearly until it is high enough for dislocations to overcome internal barriers and the pinning effect of the defects. As the dislocations propagate and interact with the defects, their interaction energy with the defect at a critical distance can cause defect absorption within the dislocation core, which, in turn, leads to a drop in the stress (Figure 5). The criterion for defect absorption is based on the condition to cause as a result of dislocation-defect interaction, the sessile $\{100\}$ loop to re-orient into a perfect glissile $\{111\}$ loop.

From Figure 5, the initial yield stress is evaluated at $0.02 \%$ offset then converted to CRSS on $\{110\}<111>$ and $\{112\}<111>$ slip planes. The average value is plotted in Figure 6 . The relationship between the defect density, $\mathrm{d}$, and CRSS, $\tau$, fits into an exponential function of the form:

$$
\tau=\tau^{*}+\beta^{*} d^{n},
$$

where $\tau^{*}$ is the CRSS in the single crystal, $\beta^{*}$ is a parameter that depends on material properties, and $\mathrm{n}$ is a constant. In this case, the three parameters values are: $\tau^{*}=90 \mathrm{MPa}, \beta^{*}=10^{-7}$, and $\mathrm{n}=0.4045$. 


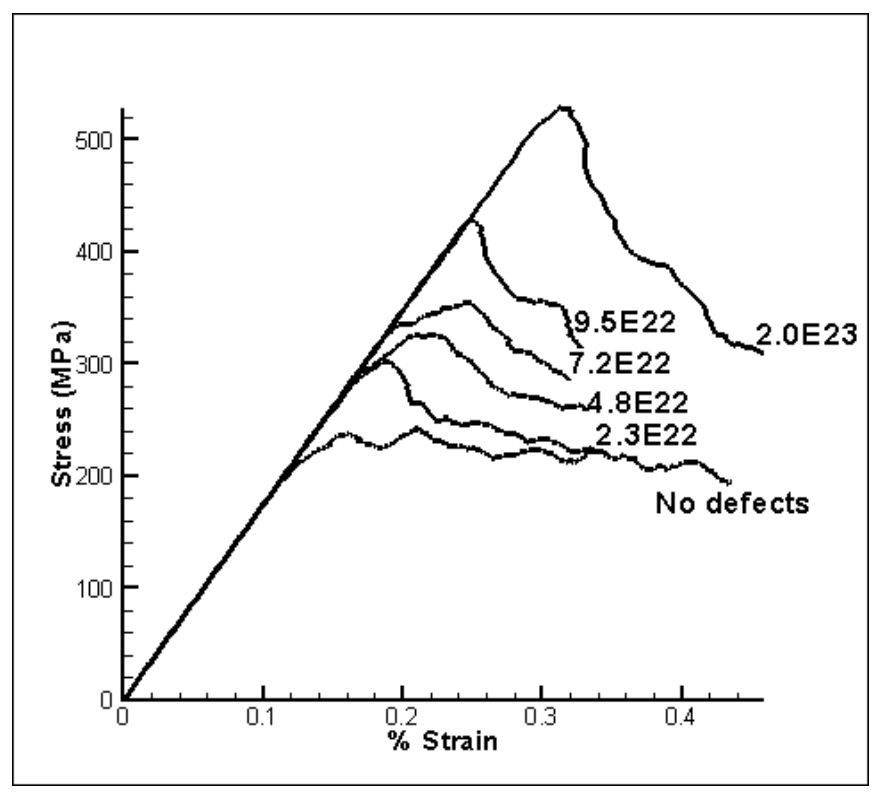

Figure 5. Predicted stress-strain curves from dislocation dynamics for various initial defect density.

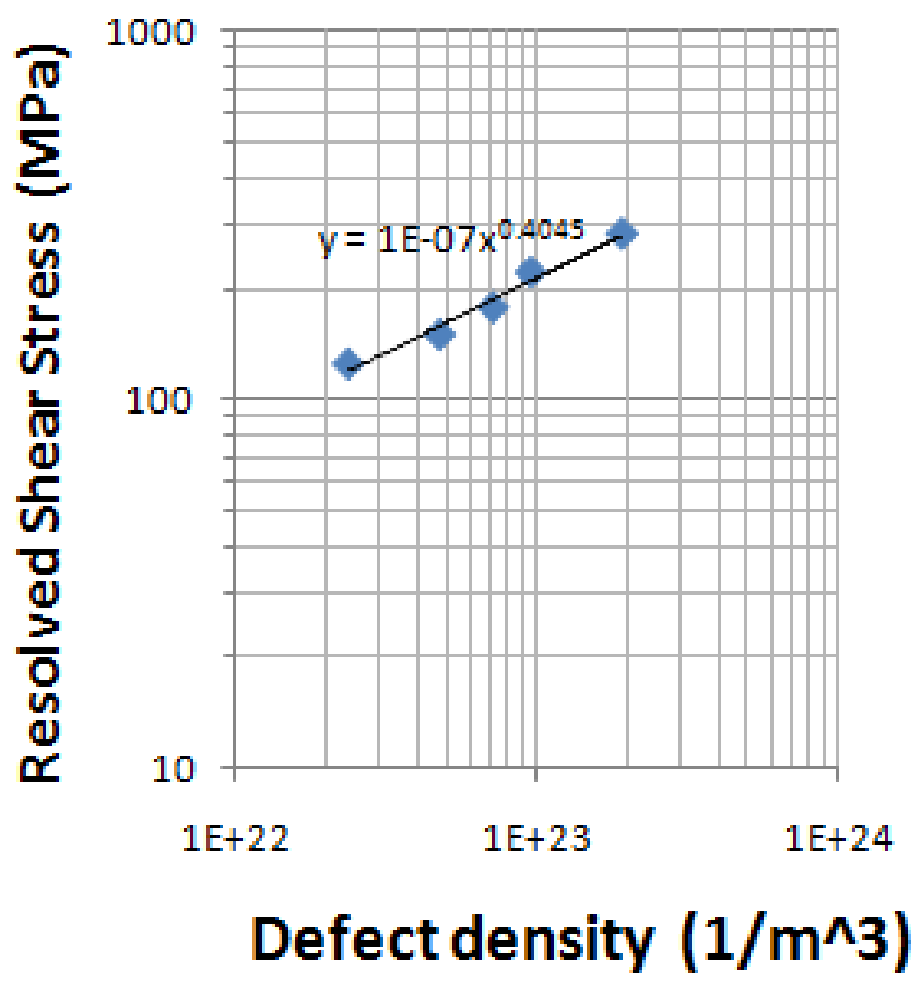

Figure 6. Resolved shear stress under different defect density predicted by dislocation dynamics models. Black line is fit to exponential function from the predicted results. 
This relationship is slightly different from the dispersed barrier model's theoretical prediction [8-10], which is based on geometrical considerations for strong obstacles intersecting the dislocation glide planes, where the yield strength increase, $\Delta \tau$, is proportional to the square root of defect cluster density, $\mathrm{d}$ :

$$
\Delta \tau=M \alpha \mu b \sqrt{d r},
$$

and where $\mathrm{M}$ is the Taylor factor, $\alpha$ is the defect cluster barrier strength, $\mu$ is the shear modulus, $b$ is the Burgers vector, and $r$ is the defect cluster size.

The resolved shear stress is passed into the viscoplasticity model to simulate the mechanical deformation behavior and texture evolution of irradiated alpha-iron. The influence of initial microstructure on the irradiation hardening was examined by investigating different textures of initial samples. In the first setup, the initial sample is composed of 500 single crystals randomly distributed. It is assumed that each crystal has the same size and volume. The texture is demonstrated in Figure 7(a) using (100), (110), and (111) pole figures. The maximum intensity in these pole figures is lower than 1.2 multiples of a random distribution. During irradiation, there is no nucleation of new crystals or crystal growth. The crystal structure of alpha-iron is assumed to remain constant as bec. Deformation mechanisms considered include three families: 12 slip systems in $\{110\}<111>, 12$ systems in $\{112\}<111>$, and 24 systems in $\{123\}<111>$. Because the activation energy in these three families is very close, their CRSS are assumed identical to the initial value as predicted from DD in Figure 4. The strain rate sensitivity of the stress is assumed to be 15 . The initial texture is random. In using the VPSC one-site version, we assumed a uniform strain path with constant strain rate and no strain hardening. After uniaxial compression along the 3 -axis to a strain of $50 \%$, the predicted texture of the deformed sample is demonstrated in Figure 7(b). In the pole figures, 3-axis is in the point of circle, 1-axis is projected east, and 2-axis is projected north. The compressed texture shows a strong alignment of (111) axis along 3-axis. Figure 7(c) shows the texture after uniaxial extension along the 3-axis to a strain of $50 \%$. It is a typical texture of bcc under tension.
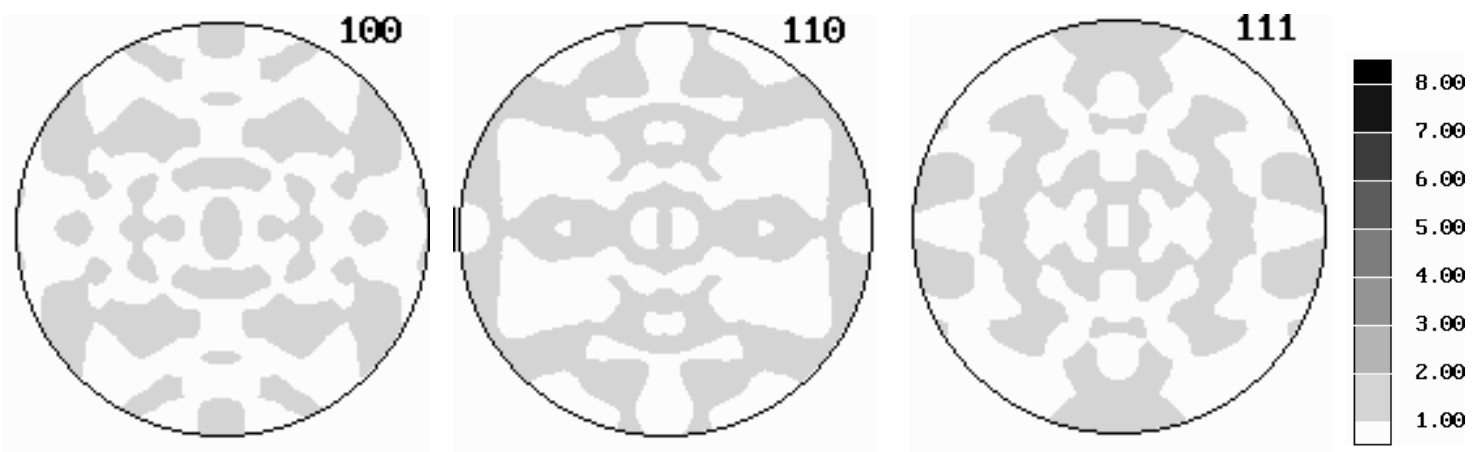

(a) 

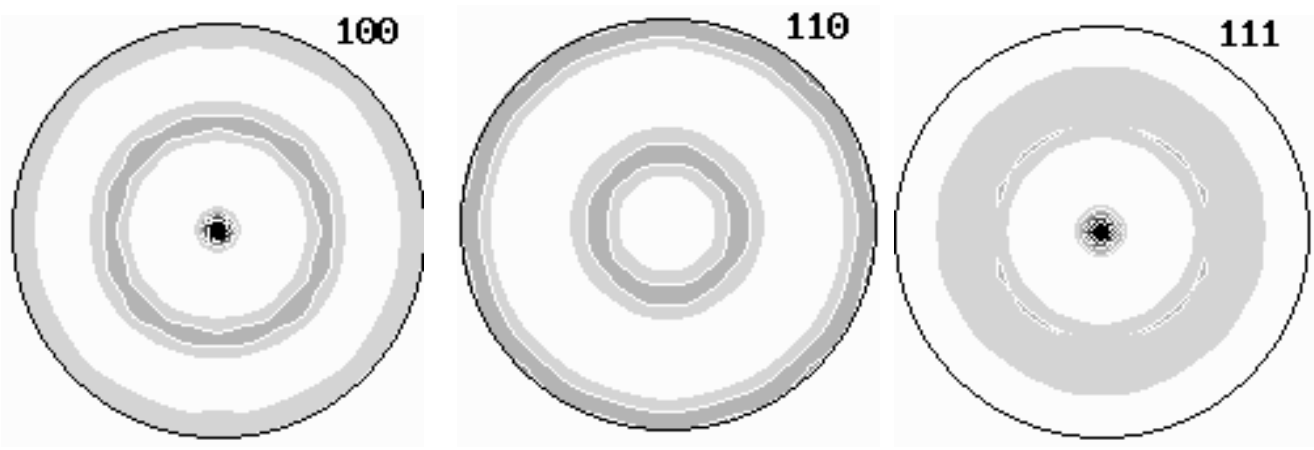

(b)
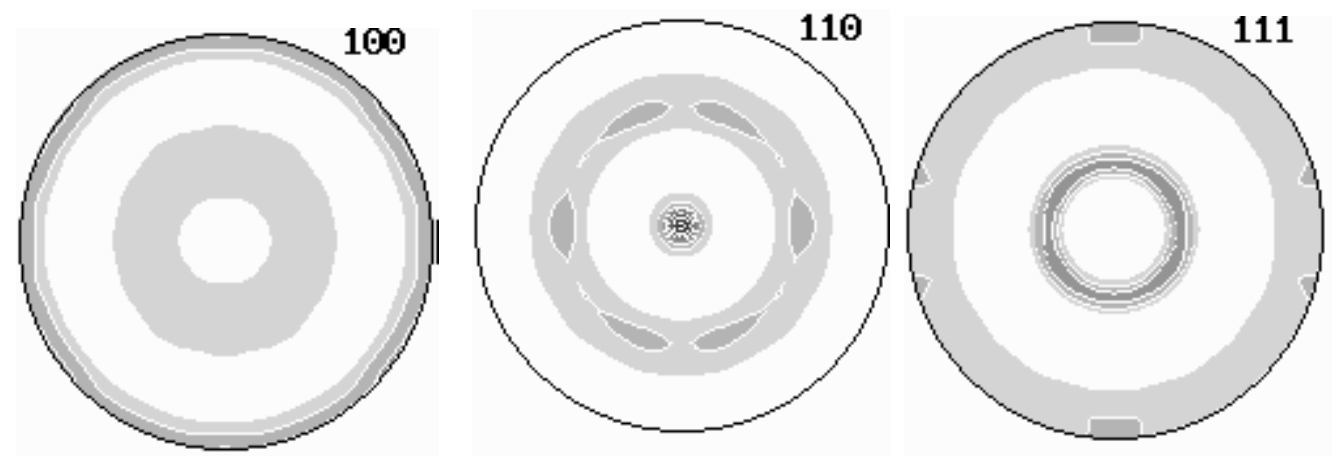

(c)

Figure 7. (100), (110), and (111) pole figures of irradiated alpha-iron with defect density of $10^{23} \mathrm{~m}^{-3}$ : (a) random sample, (b) uniaxial extended to a strain of 50\%, and (c) uniaxial compressed to a strain of 50\%.

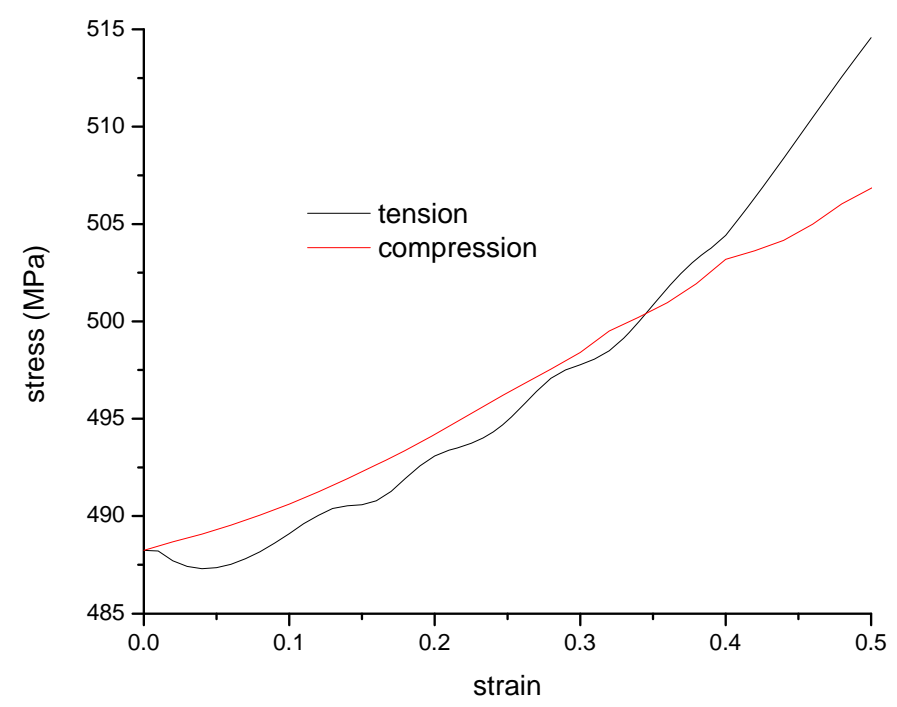

Figure 8. Predicted stress strain curve of irradiated alpha-iron with defect density of $10^{23} \mathrm{~m}^{-3} \mathrm{under}$ uniaxial tension and compression. 
The deformation behavior under uniaxial tension and compression is demonstrated in Figure 5. Strain hardening due to CRSS in Equation (4) is assumed 0. The increase of stress shown in Figure 8 is attributed to the evolution of texture.

Irradiation strengthening is shown in Figure 9 as a function of defect density, which is a function of irradiation dose. In some research, the defect density was found to be directly proportional to the irradiation dose. For single crystal configuration, such as $\{1 \overline{1} 0\}\langle 111\rangle$, the yield stress is the same as resistance-resolved shear stress. The evolution of irradiation hardening is demonstrated by a solid black curve - the same as the results shown in Figure 4. Irradiation hardening in a random textured sample is represented by blue dash curve, higher than the single crystal configuration. If all of the crystals with normal direction align with (111) direction, the yield strength will be highest (shown as a red dot curve in Figure 9). Irradiation strengthening also is the strongest in this anisotropic configuration.

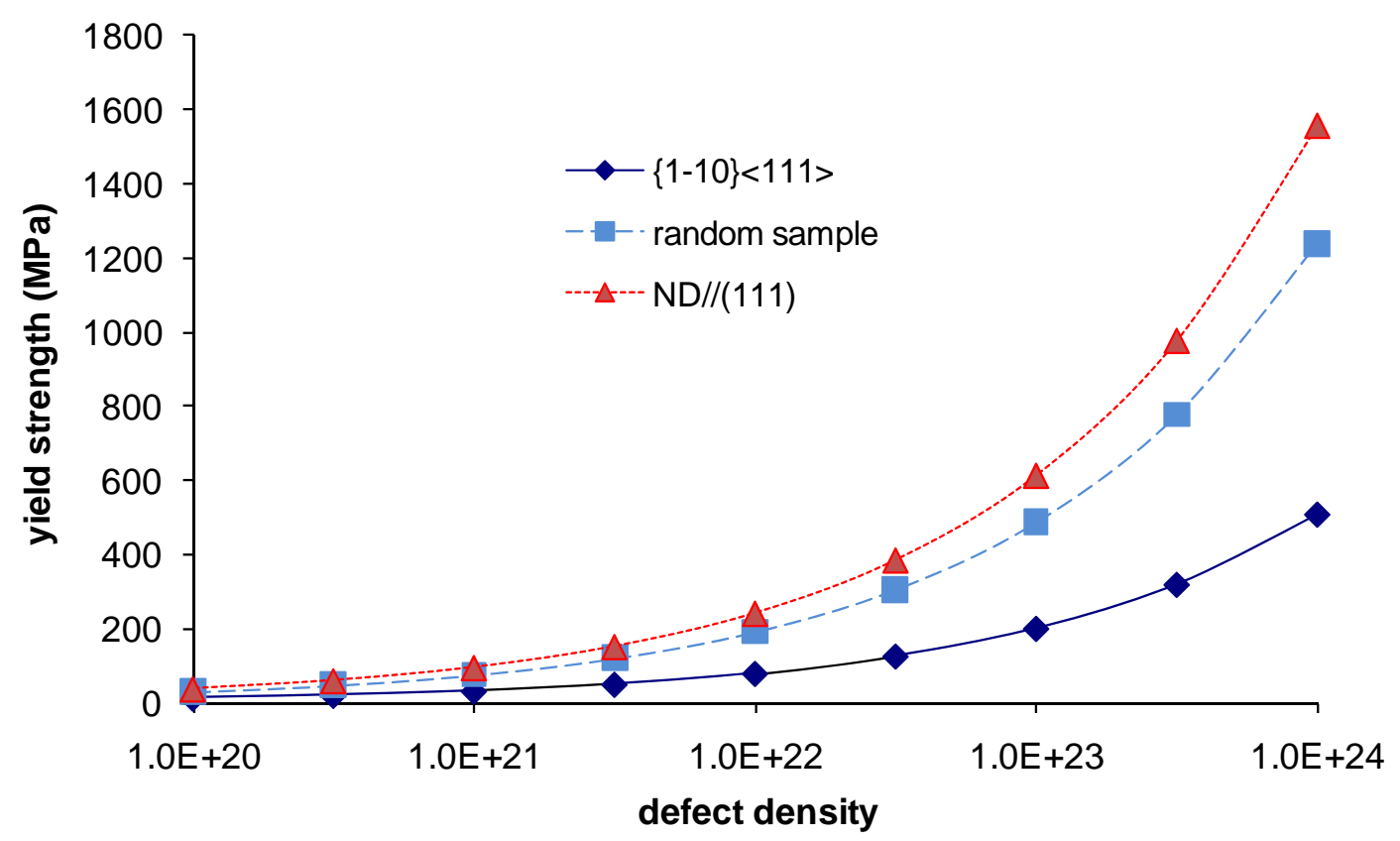

Figure 9. Predicted yield strength as a function of defect density in alpha-iron with different microstructure.

To compare directly with the experimental data on radiation strengthening, the relationship between the irradiated defect density, $d$, and irradiation dose is assumed based on previous theories and simulations. Here, the ionizing radiation absorbed dose is represented by the average number of displacements for each atom (dpa). Based on previous studies, the relationship ranges from linear one [11]:

$$
d \propto d p a
$$

to proportional to the square root of dose [12-13]:

$$
d \propto \sqrt{d p a} .
$$


Correspondingly, the relationship between the CRSS of irradiated samples, $\tau$, and irradiation dose, dpa, fits into an exponential function as:

$$
\begin{aligned}
& \tau=\tau_{0}+\beta(d p a)^{0.4045} \\
& \text { or } \tau=\tau_{0}+\beta(d p a)^{0.2022},
\end{aligned}
$$

where $\tau_{0}$ is the CRSS for unirradiated samples and $\beta$ is a parameter based on materials properties, including shear modulus and Burgers vector.

Based on different assumptions, simulation A used Equation (13a), while simulation B used Equation (13b) to study irradiation strengthening. In Figure 10, the simulation results from the multiscale model were compared with the experimental data for different stainless steel used in advanced nuclear reactors: 304L [14] (Figure 10a), 316L [14] (Figure 10b), and modified T91 [15] (Figure 10c), respectively. The original experimental data did not elaborate the condition of texture. All samples were assumed as random in this study. Before irradiation, the initial defect densities and CRSS in the samples were adjusted to reproduce the yield stress of unirradiated samples. The materials parameters used in the simulation are listed in Table 2. In Figure 10, the open symbols represent the experimental data. The filled triangle symbols of Simulation 1 are obtained using the linear relationship between the defect density and dose (Equation 11). The filled circle symbols of Simulation 2 are obtained using Equation 12. In the multiscale model, both simulations demonstrated the trend of increasing yield strength with irradiation. The assumption of a linear relationship between the defect density and dose agrees better with the experimental data in our model. However, this is only validated in the scheme of the current multiscale model, which does not account for the effect of grain boundaries.

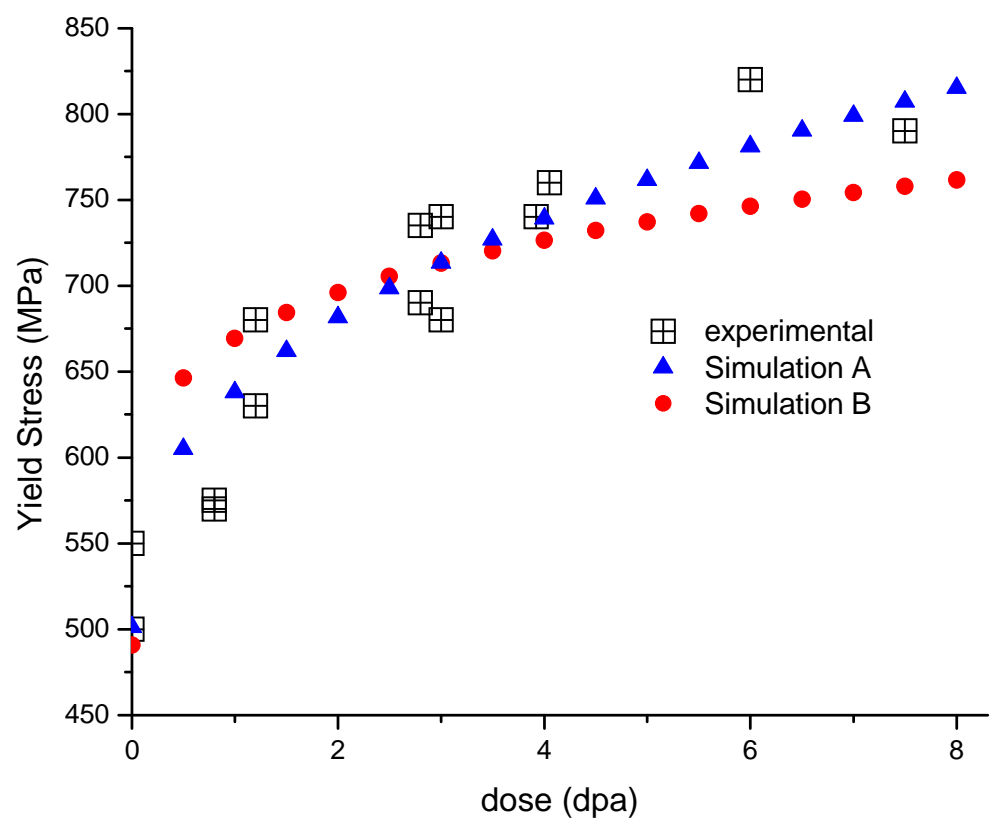

(a) 


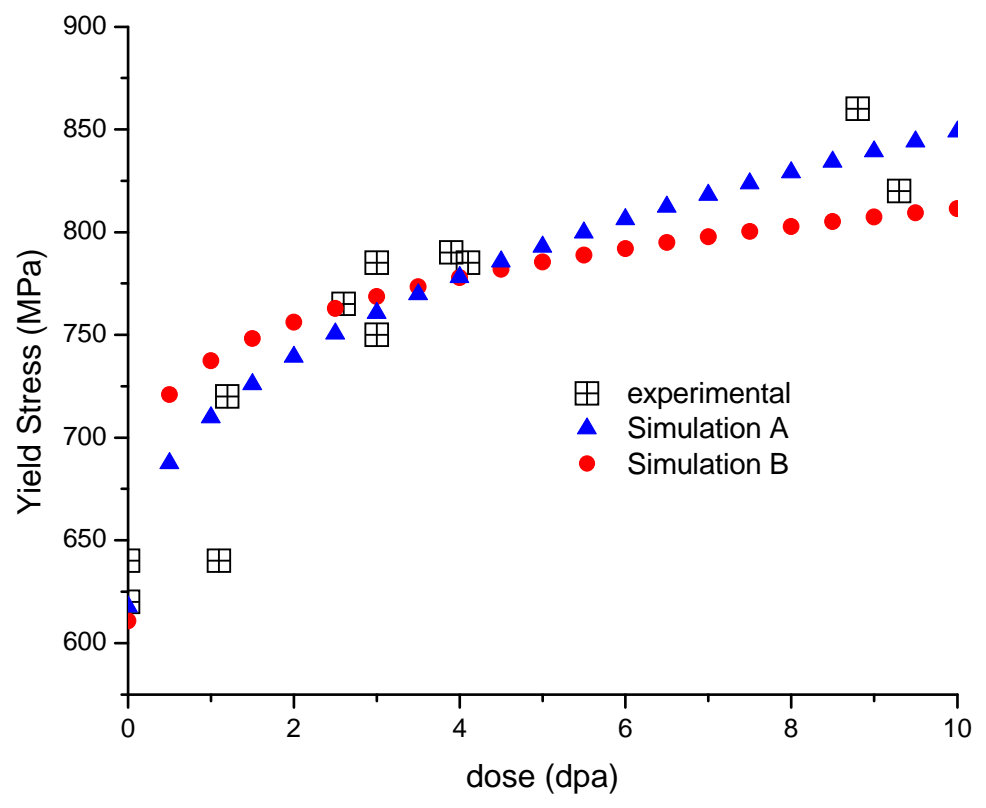

(b)

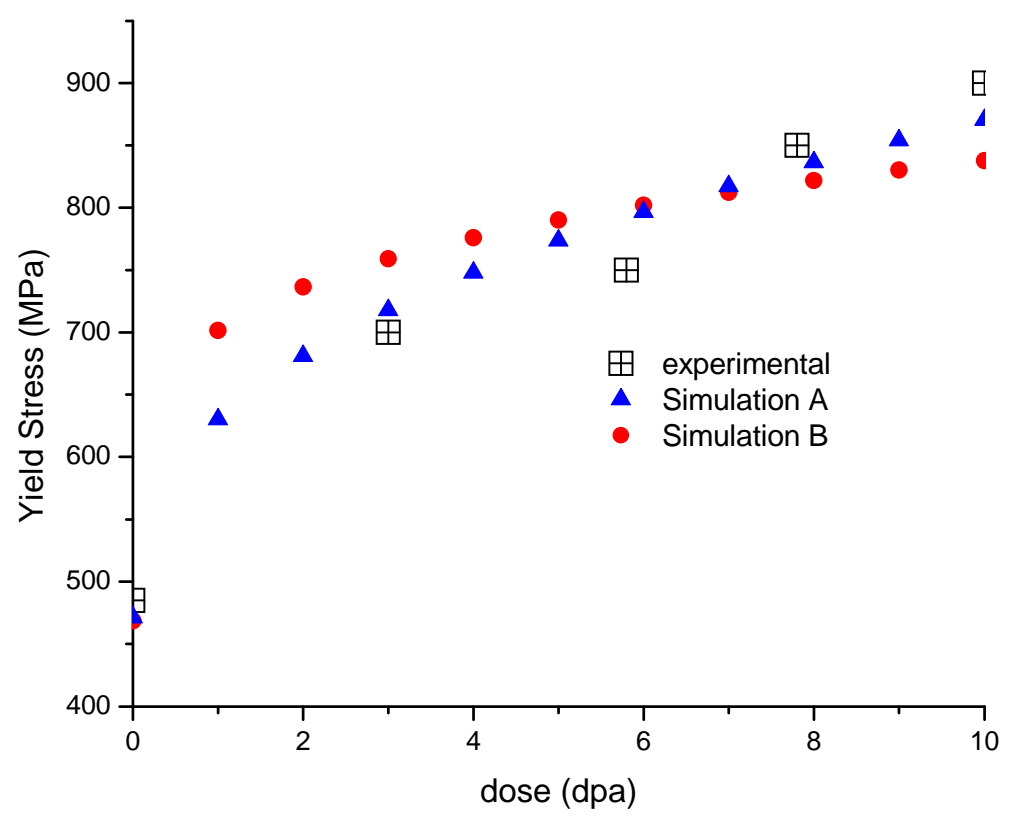

(c)

Figure 10. Variation of the yield stress with irradiation dose for stainless steel 304L (Fig.10a), 316L (Fig. 10b) and modified T91 (Fig. 10c). Experimental data (open square symbols) are compared with simulation results of filled symbols. 
Table 2: Materials parameters used in multiscale modeling the irradiation strengthening

\begin{tabular}{|c|c|c|c|c|c|c|}
\hline \multirow{2}{*}{ Samples } & \multicolumn{2}{|c|}{$316 \mathrm{~L}$} & \multicolumn{2}{c|}{$304 \mathrm{~L}$} & \multicolumn{2}{c|}{ T91 } \\
\cline { 2 - 7 } & Simulation A & Simulation B & Simulation A & Simulation B & simulation A & Simulation B \\
\hline$\tau_{0}(\mathrm{MPa})$ & 618 & 611 & 501 & 491 & 471 & 469 \\
\hline $\mathrm{B}(\mathrm{MPa})$ & 92 & 127 & 137 & 179 & 159 & 233 \\
\hline
\end{tabular}

In summary, strengthening due to irradiation is predicted considering the influence of defect density and texture. A clear trend of irradiation strengthening is predicted, and the strengthening mechanisms are proved. However, there still are many other factors not considered in this work, such as defect types and distribution, misorientation and distribution, void swelling, and migration. Introducing more factors will produce an increasingly accurate and realistic prediction of irradiation strengthening.

\section{CONCLUSIONS}

A multiscale model composed of MD, DD, and viscoplastic models was developed to predict microstructure evolution and deformation behavior of irradiated alpha-iron. The effects of defect density and texture on irradiation strengthening of alpha-iron were investigated. A parametric study was performed on strengthening due to texture. This physical-science-based prediction of an irradiation strengthening model may guide performance evaluation for future structural materials used in irradiation and other volatile environments.

\section{ACKNOWLEDGEMENTS}

This work was funded by DOE's Nuclear Energy Advanced Modeling and Simulation (NEAMS) program at Pacific Northwest National Laboratory (PNNL). PNNL is operated by Battelle for the DOE under contract No. DE-AC05-76RL01830. 


\section{REFERENCES}

[1] Deo C, Tom C, Lebensohn R, Maloy S. Modeling and simulation of irradiation hardening in structural ferritic steels for advanced nuclear reactors. Journal of Nuclear Materials 2008;377:136.

[2] Zbib HM, de la Rubia TD. A multiscale model of plasticity. International Journal of Plasticity 2002;18:1133.

[3] Khraishi TA, Zbib HM, De La Rubia TD, Victoria M. Localized deformation and hardening in irradiated metals: Three-dimensional discrete dislocation dynamics simulations. Metallurgical and Materials Transactions B-Process Metallurgy and Materials Processing Science 2002;33:285.

[4] Zbib HM, de la Rubia TD, Rhee M, Hirth JP. 3D dislocation dynamics: stress-strain behavior and hardening mechanisms in fcc and bcc metals. Journal of Nuclear Materials 2000;276:154.

[5] Lebensohn RA, Wenk HR, Tome CN. Modelling deformation and recrystallization textures in calcite. Acta Materialia 1998;46:2683.

[6] Lebensohn RA, Tome CN. A self-consistent anisotropic approach for the simulation of plasticdeformation and texture development of polycrystals- Application to Zirconium alloys. Acta Metallurgica et Materialia 1993;41:2611.

[7] Kalidindi SR, Bronkhorst CA, Anand L. Crystallographic texture evolution in bluk deformation processing of fcc metals. Journal of the Mechanics and Physics of Solids 1992;40:537.

[8] Zinkle SJ, Matsukawa Y. Observation and analysis of defect cluster production and interactions with dislocations. Journal of Nuclear Materials 2004;329:88.

[9] Kocks U. Theory of an Obstacle-Controlled Yield Strength--Report After an International Workshop. Mater. Sci. Eng. 1977;27:291.

[10] Seeger A, Diehl J, Mader S, Rebstock H. Work-hardening and work-softening of face-centred cubic metal crystals Philosophical Magazine 1957;2:323.

[11] Singh B, Evans J. Significant differences in defect accumulation behaviour between fcc and bcc crystals under cascade damage conditions. Journal of Nuclear Materials 1995;226:277.

[12] Deo C, Okuniewski M, Srivilliputhur S, Maloy S, Baskes M, James M, Stubbins J. Helium bubble nucleation in bcc iron studied by kinetic Monte Carlo simulations. Journal of Nuclear Materials 2007;361:141.

[13] Deo C, Okuniewski M, Srivilliputhur S, Maloy S, Baskes M, James M, Stubbins J. The effects of helium on irradiation damage in single crystal iron. Journal of Nuclear Materials 2007;367:451.

[14] Maloy SA, James MR, Willcutt G, Sommer WF, Sokolov M, Snead LL, Hamilton ML, Garner F. The mechanical properties of 316L/304L stainless steels, Alloy 718 and Mod 9Cr-1Mo after irradiation in a spallation environment. Journal of Nuclear Materials 2001;296:119.

[15] Dai Y, Jia X, Farrell K. Mechanical properties of modified 9Cr-1Mo (T91) irradiated at $300^{\circ} \mathrm{C}$ in SINQ Target-3. Journal of Nuclear Materials 2003;318:192. 